\section{Expression of acetylated tubu- lin in the postnatal developing mouse cochlea}

\author{
Wen Jing Liu, ${ }^{1,2}$ Chuan Xi Wang, ${ }^{1}$ \\ Hao Yu, ${ }^{3}$ Shao Feng Liu, ${ }^{1}$ Jun Yang ${ }^{2}$ \\ ${ }^{1}$ Department of Otorhinolaryngology- \\ Head and Neck Surgery, Yijishan \\ Hospital, Wannan Medical College, \\ Wuhu \\ ${ }^{2}$ Department of Otorhinolaryngology- \\ Head and Neck Surgery, Xinhua \\ Hospital, Shanghai Jiaotong University \\ School of Medicine \\ ${ }^{3}$ Anhui Province Key Laboratory of \\ Biological Macro-Molecules Reserch, \\ Wannan Medical College, Wuhu, China
}

\section{Abstract}

Microtubules are an essential component of the cytoskeleton of a eukaryotic cell. The post-translational tubulin modifications play an important role in regulating microtubule properties, acetylation tubulin is one of the major post-translational modifications of microtubules. Acetylation tubulin has also been shown to be expressed in the cochlea. However, the detailed expression profiles of acetylation tubulin protein during development have not yet been investigated in the postnatal mammalian cochlea. Here, we first examined the spatio-temporal expression of acetylated tubulin in the mouse cochlea during postnatal development. At postnatal day 1 (P1), acetylated tubulin was localized primarily to the auditory nerve inside the cochlea and their synaptic contacts with the inner and outer hair cells (IHCs and OHCs). In the organ of Corti, acetylated tubulin occurred first at the apex of pillar cells. At P5, acetylated tubulin first appeared in the phalangeal processes of Deiters' cells. At P8, staining was maintained in the phalangeal processes of Deiters' cells and neural elements. At P10, labeling in Deiters' cells extended from the apices of OHCs to the basilar membrane, acetylated tubulin was expressed throughout the cytoplasm of inner and outer pillar cells. At P12, acetylated tubulin displayed prominent and homogeneous labeling along the full length of the pillar cells. Linear labeling was present mainly in the Deiters' cell bodies underlying OHCs. Between P14 and P17, acetylated tubulin was strongly expressed in inner and outer pillar cells and Deiters' cells in a similar pattern as observed in the adult, and labeling in these cells were arranged in bundles. In addition, acetylated tubulin was expressed in stria vascularis, root cell bodies, and a small number of fibrocytes of the spiral ligament until the adult. In the adult mouse cochlea, immunostaining continued to predominate in Deiters' cells and pillar cells. In Deiters' cells, immunolabeling formed cups securing OHCs basal portions, and continued presence of acetylated tubulin-labeled nerve terminals below IHCs was shown. Our results presented here underscored the essential role played by acetylated tubulin in postnatal cochlear development, auditory neurotransmission and cochlear mechanics.

\section{Introduction}

Microtubules, heterodimers of $\alpha$ - and $\beta$ tubulin, are implicated in multiple roles including intracellular transport, maintenance of cell morphology, and formation of cell polarity. ${ }^{1,2}$ Tubulin, the building block of microtubules, is subject to post-translational modifications proposed to specify microtubule subpopulations for particular functions. ${ }^{3,4}$ Changes in tubulin post-translational modifications have been associated with neurodegenerative disorders, cancer, heart diseases and other pathological conditions. ${ }^{5,6}$ Stable microtubules are well known to contain acetylated tubulin, which is one of the major post-translational modifications of microtubules. Acetylation tubulin has been implicated in the differentiation of microtubule structure and function. ${ }^{7,8}$ In the rodent cochlea, previous studies have shown that expression of acetylated tubulin played significant roles in auditory function. For example, the intensity of staining of acetylated tubulin correlated with microtubule stability in supporting cells of the gerbil auditory sensory epithelium. ${ }^{9}$ Alteration of thyroid hormones led to a decrease in acetylated tubulin immunofluorescence in supporting pillar and Deiters' cells, and disrupted cell mechanics in the developing organ of Corti. ${ }^{10}$ More recently, a report demonstrated that the reduction in acetylated $\alpha$-tubulin labeling in the pillar cells in early postnatal stages was linked to mechanism of gjb2-related hearing loss. ${ }^{11} \mathrm{In}$ the mammalian cochlea, the widespread expression of acetylated tubulin at different stages has been obtained, suggesting its expression in normal cochlea was related to the development. ${ }^{12,13}$ However, the detailed and dynamic expression pattern of acetylation tubulin during cochlear development is not still determined, which is essential for understanding its potential roles in cochlear development and hearing. In the present study, we investigate the spatio-temporal expression of acetylated tubulin in the mouse cochlea during postnatal development in more detail by immunofluorescence
Correspondence: Jun Yang, Department of Otorhinolaryngology-Head \& Neck Surgery, Xinhua Hospital, Shanghai Jiaotong University School of Medicine, Shanghai Jiaotong University Ear Institute, Shanghai Key Laboratory of Translational Medicine on Ear and Nose diseases (No. 14DZ2260300), Shanghai 200092, China.

Tel. +86.21.25078532 - Fax: +86.21.65156489.

E-mail: yangjun@xinhuamed.com

Shao Feng Liu, Department of Otorhinolaryngology-Head and Neck Surgery, Yijishan Hospital, Wannan Medical College, Wuhu, China. E-mail: liusf_cn@163.com

Key words: Acetylated tubulin; immunofluorescence; expression; mouse; cochlea; development.

Contributions: WJL, CXW, HY contributed equally to this work; SFL, JY, defined the research theme, designed methods and revised the manuscript. All authors approved the version to be published.

Conflict of interest: The authors declare no potential conflict of interest.

Funding: This work was supported by the National Natural Science Foundation of China (No.81170919, No. 81470689), the project of Shanghai Municipal Science and Technology Commission (No. 14DJ1400201, No. 14DZ2260300).

Received for publication: 8 May 2018. Accepted for publication: 1 August 2018.

This work is licensed under a Creative Commons Attribution-NonCommercial 4.0 International License (CC BY-NC 4.0).

(C) Copyright W.J. Liu et al., 2018

Licensee PAGEPress, Italy

European Journal of Histochemistry 2018; 62:2942 doi:10.4081/ejh.2018.2942

analysis. Images are acquired by confocal laser-scanning microscopy and analyzed semi-quantitatively by software. This is the first study showing a comprehensive immunohistochemical mapping of acetylated tubulin in the developing mouse cochlea across ten postnatal time points, providing an evidence that acetylated tubulin has an important role in postnatal mouse cochlear development.

\section{Materials and Methods}

\section{Animals}

Male and female BALB/c mice of the following ages were examined in this study, namely, postnatal day 1 (P1), P5, P8, P10, P12, P14, P17, P21, P30 and the adult 

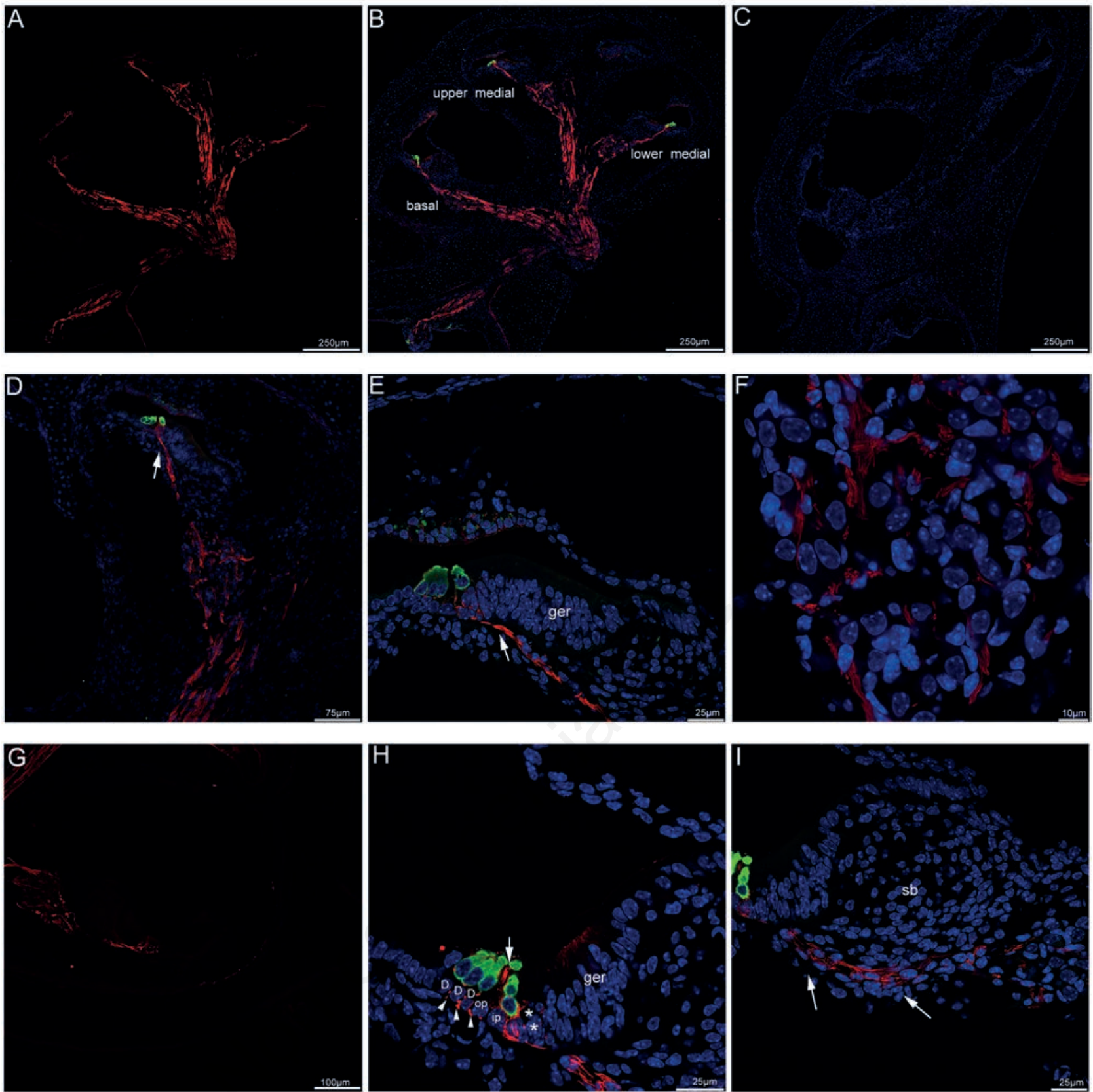

Figure 1. Acetylated tubulin immunolabeling in the mouse cochlea at P1. A) A low-magnification view of the entire cochlear section labeled with acetylated tubulin (red). The antibody against acetylated tubulin labeled tracts where the auditory nerves and vestibular nerves traveled inside the cochlea. B) A low-magnification view of the entire cochlear section double-labeled with acetylated tubulin (red) and myosin VIIa (green, hair cells marker) and the merged image+DAPI. C) The absence of acetylated tubulin and myosin VIIa immunofluorescence in negative control at P1, omitting the primary antibody. Cell nuclei were counterstained with DAPI (blue). D) An overview of acetylated tubulin (red) and myosin VIIa (green) labeling in the upper medial turn of the mouse cochlea at P1 and the merged image +DAPI. The auditory nerves in Rosenthal's canal in the modiolus close to the apical turn showed significant immunoreactivity for acetylated tubulin (red), noted that the radial peripheral process (arrow) of the auditory nerve projected toward the region of myosin VIIa-labelled hair cells (green). E) Detail of acetylated tubulin (red) labeling in the organ of Corti in the upper medial turn of P1. Immunoreactivity in the peripheral process (arrow) of the auditory nerve reached the inner and outer hair cells. Cell nuclei were counterstained with DAPI (blue). F) Detail of acetylated tubulin (red) labeling in the Rosenthal's canal of the upper medial turn of P1, noted that lack of immunolabeling in the cell bodies of spiral ganglion neurons located within Rosenthal's canal. Cell nuclei were counterstained with DAPI (blue). G) An overview of acetylated tubulin (red) labeling in the lower medial turn of the mouse cochlea at P1. H) Detail of acetylated tubulin (red) and myosin VIIa (green) labeling in the organ of Corti in the basal turn at P1. Deiters' cells were identified by the position of their nuclei close to the basilar membrane, acetylated tubulin-positive fibers and terminals (asterisks) were observed beneath inner hair cells, acetylated tubulin expression was found as punctate immunopositive profiles (arrowheads) between Deiters' cells. Note that acetylated tubulin immunolabeling appeared in the apex of pillar cell (large arrow) between inner and outer hair cells. Cell nuclei were counterstained with DAPI (blue). I) In the basal turn of P1, acetylated tubulin-labeled nerve fibers (arrows) extended to the inner hair cells. Cell nuclei were counterstained with DAPI (blue). D, Deiters' cell; ger, greater epithelial ridge; sb, spiral limbus; op, outer pillar cell; ip, inner pillar cell. 
$\left(\mathrm{P} 30^{+}\right)$. All procedures of the present study had approval from the Animal Use and Care Committee of Wannan Medical College.

\section{Tissue preparation}

The detailed methods and procedures of immunofluorescent staining could be found in our previous reports in rat cochlear tissue. ${ }^{16,17}$ Briefly, post-natal mouse at different developmental stages (P1, P5, P8, P10, $\mathrm{P} 12, \mathrm{P} 14, \mathrm{P} 17, \mathrm{P} 21, \mathrm{P} 30$ and $\mathrm{P} 30^{+}$were fixed initially by intracardiac perfusion with ice-cold $4 \%$ paraformaldehyde in $0.1 \mathrm{M}$ phosphate buffer ( $\mathrm{pH}$ 7.4) and the cochlea was then quickly removed. The round and oval windows of the cochlea were opened, and a small hole made in the bony apex of the cochlea. After perilymphatic perfusion with the above fixative, cochlea was post- fixed in the same fixative for $35 \mathrm{~min}$ at room temperature. P5 and older animals were decalcified in $10 \%$ EDTA at $\mathrm{pH}$ 7.4. One should note that decalcification time for mice cochlea tissue needed to be further optimized. In our protocol, P30 mouse cochlea was decalcified in 10\% EDTA solution at $4^{\circ} \mathrm{C}$ for $16 \mathrm{~h}$, and excess tissue surrounding the cochlea was removed every three hours. The cochleae were subsequently placed in a gradient of sucrose $15 \%$ for $3 \mathrm{~h}$ and $30 \%$ overnight. The cochleae were then embeded in optimum cutting temperature compound (OCT) at $4^{\circ} \mathrm{C}$ for $3 \mathrm{~h}$. Serial 7 $\mu \mathrm{m}$-thick sections were cut with a Leica cryostat microtome.

\section{Antibodies}

A monoclonal mouse anti-acetylated tubulin antibody (Clone 6-11B-1, Cat. No. T6793, Sigma-Aldrich, St. Louis, MO, USA) was used to detect acetylated tubulin. This antibody has been used to detect acetylated $\alpha$-tubulins from many organisms including mouse, ${ }^{18,19}$ and detailed information about the specificities of this antibody is accessible in the literation. ${ }^{20} \mathrm{~A}$ polyclonal rabbit anti-myosin VIIa antibody (Cat. No. 25-6791, Proteus Biosciences, Ramona, CA, USA) was used to label HCs.

\section{Immunofluorescence}

Cochlear cross-sections were treated for 30 min with $10 \%$ donkey serum and $0.3 \%$ Triton X-100 in PBS in order to make membranes more permeable to antibodies and prevent nonspecific binding of the primary antibody. Then sections were incubated
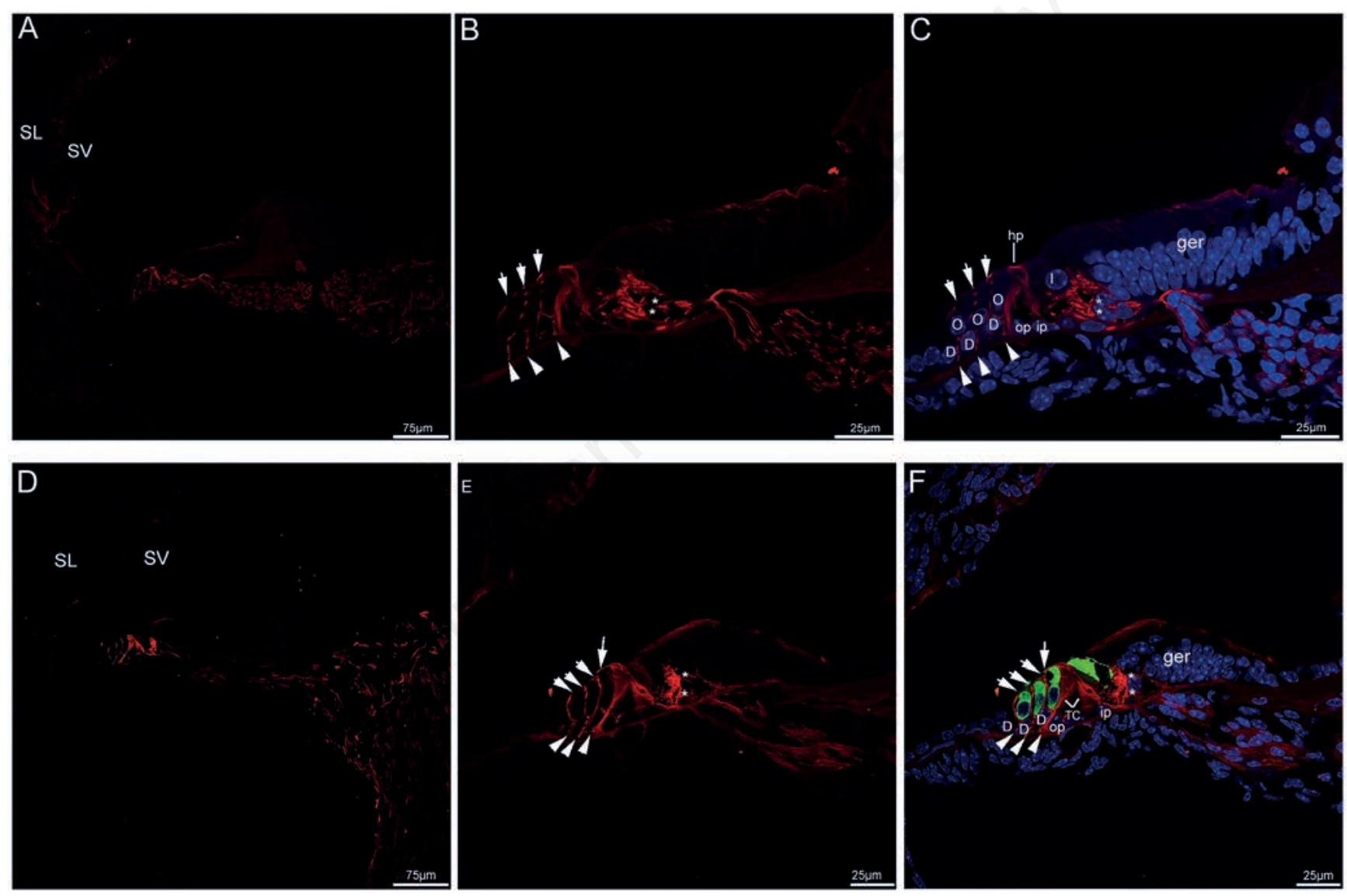

Figure 2. Acetylated tubulin immunolabeling in the mouse cochlea at P5 and P8. A) An overview of acetylated tubulin (red) labeling in the medial turn of the mouse cochlea at P5. B,C) Detail of acetylated tubulin (red) labeling in the organ of Corti in the medial turn of P5. The neural plexus (asterisks) beneath the inner hair cell showed acetylated tubulin immunolabeling, three discrete rows of fibers (arrowheads) under the outer hair cell stained positively for acetylated tubulin. Note that the phalangeal processes (small arrows) of premature Deiters' cells that lied between outer hair cells began to express acetylated tubulin, moderate immunolabeling was seen in the head plates of the pillar cells. Cell nuclei were counterstained with DAPI (blue). D) An overview of acetylated tubulin (red) labeling in the medial turn of the mouse cochlea at P8. Acetylated tubulin (red) labeling seemed to be limited to the organ of Corti. E,F) Detail of acetylated tubulin (red) labeling and myosin VIIa (green) labeling in the organ of Corti in the medial turn of P8 and the merged image +DAPI. The tunnel of Corti opened up between most of the lower portions of inner and outer pillar cells, acetylated tubulinlabeled nerve terminals (asterisks) became more clustered around the base of the inner hair cells, acetylated tubulin (red) labeling was also observed three discrete rows of fibers (arrowheads) under the outer hair cell. Acetylated tubulin-labeled the phalangeal processes of Deiters' cells (small arrows) filled the spaces between the outer hair cells. Noted that a process (large arrow) was extended by the inner pillar cell and expressed acetylated tubulin. D, Deiters' cell; I, inner hair cell; O, outer hair cell; TC, tunnel of Corti; ger, greater epithelial ridge; hp, head plate; op, outer pillar cell; ip, inner pillar cell; SL, spiral ligament; SV, stria vascularis. 
overnight at $4{ }^{\circ} \mathrm{C}$ with two primary antibodies: 1:250 monoclonal mouse anti-acetylated tubulin antibodies (Sigma-Aldrich) and 1:250 polyclonal rabbit anti-Myosin VIIa (Proteus Biosciences). After rinsing three times for $15 \mathrm{~min}$ in the $0.01 \mathrm{M}$ PBS, sections were put for $1 \mathrm{~h}$ at $37^{\circ} \mathrm{C}$ in the mixture of the secondary antibodies labeled with Alexa 488-conjugated donkey anti-rabbit (1:200; Jackson Labs, Bar Harbor, ME, USA) and NL637-conjugated donkey anti-mouse antibodies (1:200; RD) secondary antibodies. Negative controls were established by omitting the primary antibody. Sections were rinsed, counterstained with 4,6-diamidino2-phenylindole (DAPI; 1:600; Biyuntian,
China) for $5 \mathrm{~min}$ to visualize cell nuclei. Following washing with PBS, fluorescence was preserved by sealing specimens with a solution of equal parts PBS and glycerol. Cryostat sections were viewed with a LeicaSP8 laser scanning confocal microscope with 10x $(\mathrm{NA}=0.4), 20 \mathrm{x} \quad(\mathrm{NA}=0.7), 40 \mathrm{x}$ $(\mathrm{NA}=0.85)$ and $63 \mathrm{x}(\mathrm{NA}=1.4)$ oil-immersion objectives, equipped with 458,488 , and $514 \mathrm{~nm}$ multiline argon; $561 \mathrm{~nm}$ helium neon green; and $633 \mathrm{~nm}$ helium neon red lasers. LAS AF Version 3.2.1.9702 acquisition software was used. From P8 and onwards, images were a stack of two to four Z-planes. For immunofluorescence quantification experiments the same settings for each condition and throughout experiments were used. Immunostaining presented in figures was representative of two individual experiments. Images were cropped and resized using Adobe Photoshop CS version 8 (Adobe, Seattle, WA, USA)

\section{Immunofluorescence quantification and statistical analysis}

For semi-quantitative analysis of acetylated tubulin immunolabeling intensity in the specific regions of mouse cochlea sections at various time points tested, the computer image analysis software Image $J$ was performed (freely downloadable from the Image $\mathrm{J}$ website). The digital image (cap-
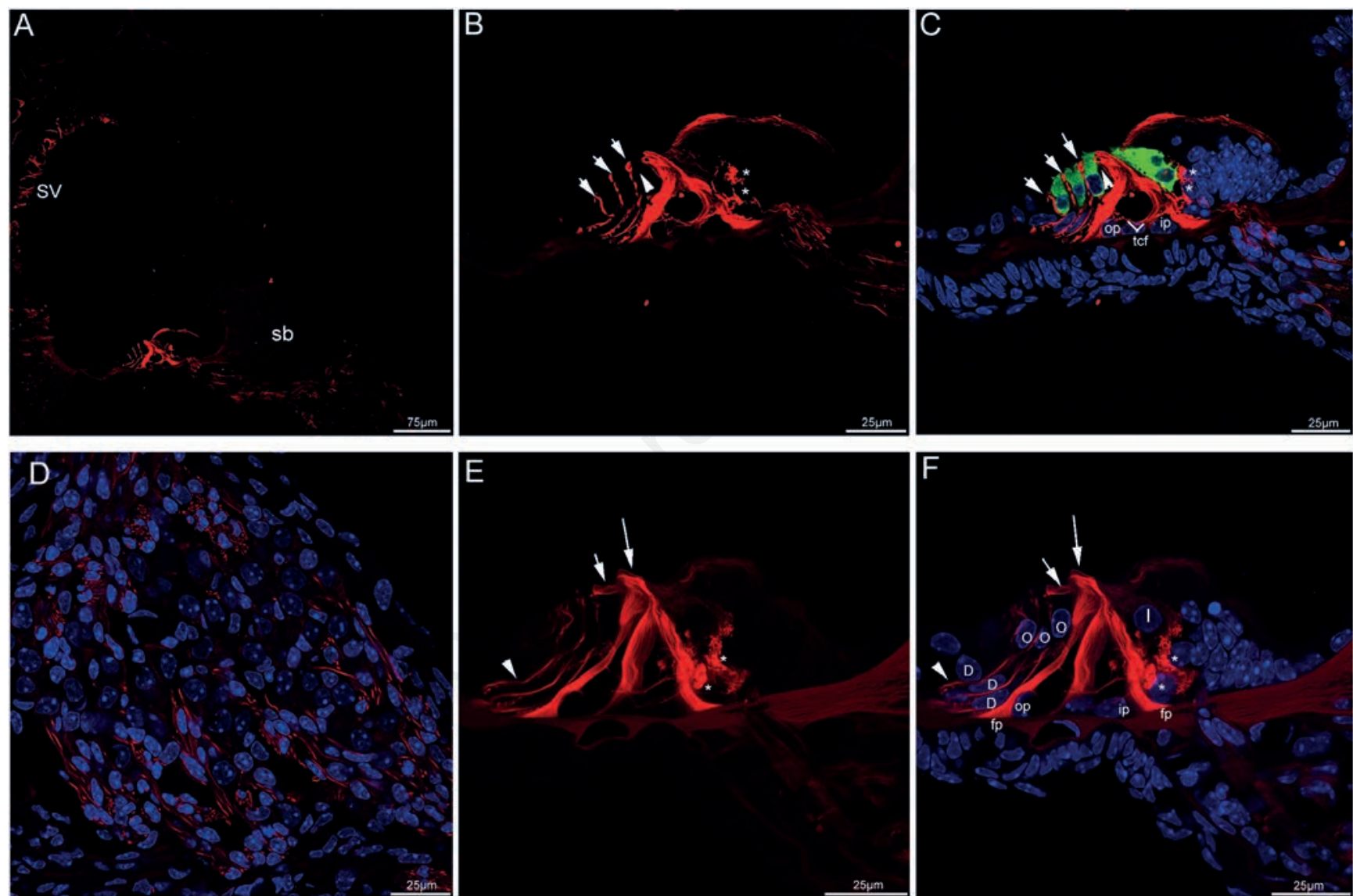

Figure 3. Acetylated tubulin immunolabeling in the mouse cochlea at P10 and P12. A) An overview of acetylated tubulin (red) labeling in the medial turn of the mouse cochlea at P10. B,C) Detail of acetylated tubulin (red) labeling and myosin VIIa (green) labeling in the organ of Corti in the medial turn of P10 and the merged image +DAPI. Nuel's space (arrowhead) opened. The neural plexus (asterisks) beneath the IHCs showed strong acetylated tubulin immunolabeling, immunostaining for acetylated tubulin was seen throughout the cell body of inner and outer pillar cells. Noted that in Deiters' cells, acetylated tubulin expression ran in a longitudinal line, punctuated by small negative areas. Immunolabeling extended from the apical portion of each outer hair cells (arrows) toward the basilar membrane. Positive immunostaining was also detected in the tunnel crossing fibers. D) Detail of acetylated tubulin (red) labeling in the Rosenthal's canal of the medial turn of P10. Immunostaining of the auditory nerve fibers was maintained. Cell nuclei were counterstained with DAPI (blue). E,F) Detail of acetylated tubulin (red) labeling in the organ of Corti in the medial turn of P12. The labeling for acetylated tubulin was intense and uniform along the full length of the inner and outer pillar cells, its expression in the inner pillar cell phalangeal processes (large arrow) was pronounced. There was moderate labeling in the apical region of the outer pillar cell, the beam bundle (small arrow) interdigitating between the outer hair cells showed acetylated tubulin immunolabeling. Noted that the foot plates of both cell types showed strong labeling. Linear labeling was uniform along most length of Deiters' cells and was present mainly in the Deiters' cell bodies (arrowhead), which spanned the distance between the base of the outer hair cells and the basilar membrane. Strong immunofluorescence was clustered in the basolateral region of the inner hair cells (asterisks). Merged image with cell nuclei stained with DAPI (blue). tcf, tunnel crossing fibers; fp, footplate; sb, spiral limbus; D, Deiters' cell; I, inner hair cell; O, outer hair cell; op, outer pillar cell; ip, inner pillar cell; SV, stria vascularis. 
tured as described above) was converted to 8 bit. The threshold for staining detection was set, and the immunostained area of interest was manually drawn by using the polygon tool. In the menu options selected Analyze > Measure to calculate the area, integrated density (IntDen), and mean intensity of the region of interest. Values were obtained from two cochlear sections per group and all statistical analysis were performed with IBM SPSS Software, ver. 20.0 (IBM Co., Armonk, NY, USA). The data are presented as the mean \pm standard deviation. Statistical significance was determined using a one-way analysis of variance, and a least significant difference posthoc test was used to evaluate the statistical differences between groups. Differences were considered statistically significant when the P-value was less than 0.05 .

\section{Results}

Developmental expression of acetylated tubulin in mouse cochlea at P1, P5, P8, P10 and P12

In the present study, the spatio-temporal expression of acetylated tubulin was examined by immunofluorescence on cryosections of the mouse cochlea at various developmental stages. At P1, labeling was mainly limited to the auditory nerve and their terminals innervating the organ of Corti (Figure1 A,B). These results were consistent with prior data demonstrating that the distribution of nerve fibers was labeled with a monoclonal antibody to acetylated tubulin in the $\mathrm{P} 0$ mouse cochlea, which permitted visualization of the innervation pattern. ${ }^{21,22}$ No positive immunolabeling was observed in the negative control (Figure 1C). To determine the precise localization of acetylated tubulin in the organ of Corti, we performed double labeling with acetylated tubulin and an antibody to myosin VIIa, a known marker of developing sensory hair cells. In the medial turn of P1, acetylated tubulin-labeled radial fibers projected to the base of IHCs and OHCs, which formed synaptic contacts with the HCs, while acetylated tubulin was rarely observed in the cell bodies of spiral gan-
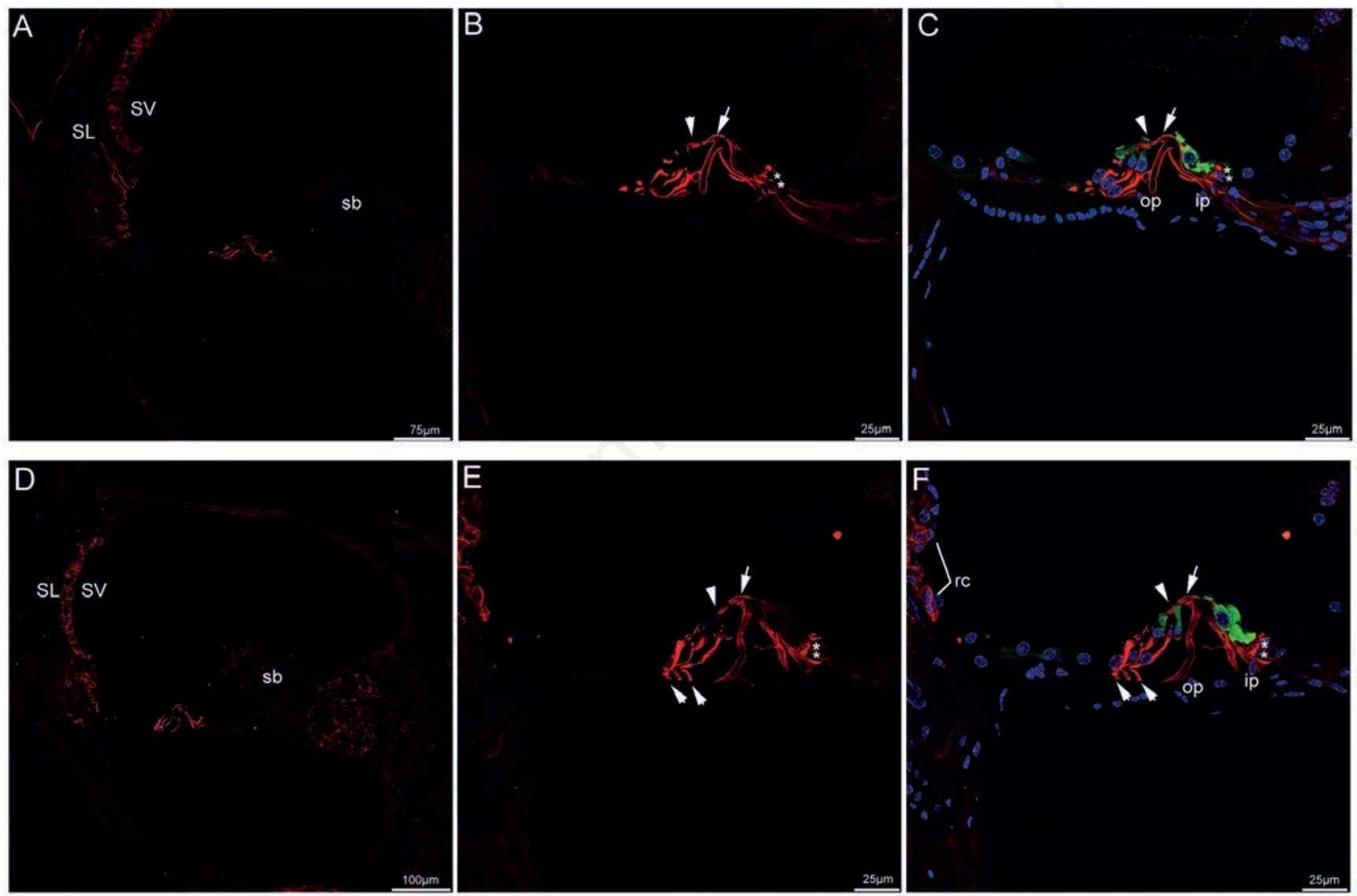

Figure 4. Confocal images of acetylated tubulin (red) and myosin VIIa (green) immunolabeling in the mouse cochlea at P14, P17 and the merged image +DAPI. A) An overview of acetylated tubulin labeling in the medial turn of the mouse cochlea at P14. Noted that stria vascularis and a small number of fibrocytes of the spiral ligament exhibited moderate immunostaining. B,C) Detail of acetylated tubulin labeling in the organ of Corti in the medial turn of P14 and the merged image +DAPI. Immunostaining in the pillar cells were arranged in parallel bundles, acetylated tubulin-labeled bundles ran the full length of the inner and outer pillar cells, especially throughout the transcellular bundle (arrow) at apical surface of inner pillar cell and the beam bundle (arrowhead) in the outer pillar cell. Noted that acetylated tubulin immunostaining in three rows of Deiters' cells became thicker and formed bundles. Punctate acetylated tubulin immunostaining was evident around the base of the inner hair cells (asterisks). D) An overview of acetylated tubulin labeling in the medial turn of the mouse cochlea at P17. E,F) Detail of acetylated tubulin labeling in the organ of Corti in the medial turn of P17. Acetylated tubulin continued to be expressed in the pillar cells, three rows of Deiters' cells and nerve terminals (asterisks) under the inner hair cells. Acetylated tubulin was also present in the beam bundle (arrowhead) in the outer pillar cell and transcellular bundle (large arrow) at apical surface of inner pillar cell. Noted that Deiters' feet (small arrows) contacted the basilar membrane, root cells showed positive expression. Cell nuclei were counterstained with DAPI (blue). SL, spiral ligament; SV, stria vascularis; rc, root cells; sb, spiral limbus; op, outer pillar cell; ip, inner pillar cell. 
glion neurons in the Rosenthal's canal (Figure $1 \mathrm{D}-\mathrm{G})$. The neural staining in the synaptic area underneath the IHCs and OHCs was evident in the basal turn (Figure $1 \mathrm{H}-\mathrm{I})$. At the medial turn of P5, acetylated tubulin showed moderate expression in the head plate of the pillar cells and outer pillar cell. Labeling for acetylated tubulin was observed as dense nerve plexus beneath IHCs and the three rows of nerve fibers under the OHCs. Interestingly, acetylated tubulin first appeared in the phalangeal processes of Deiters' cells, between the base of the OHCs and the reticular lamina (Figure 2 A-C). As development proceeded, the opening of the tunnel of Corti between the inner and outer pillar cells was observed at P8, and Nuel's space between outer pillar cells and OHCs was formed at P10. These are important hallmarks in cochlear devel- opment. ${ }^{23}$ During this period, changes in the extent of labeling across the pillar and Deiters' cells were observed at two key developmental stages. At P8, the apical surface of inner pillar cell extended a process and showed positive labeling. Labeling for acetylated tubulin was maintained in the phalangeal processes of Deiters' cells, both the inner and outer pillar cells exhibited moderate staining (Figure $2 \mathrm{D}-\mathrm{F}$ ). At P10, labeling for acetylated tubulin in Deiters' cells was more extensively labeled than at P8, a slightly interrupted line extended from the reticular lamina toward the basilar membrane, more intense staining was distributed throughout the cytoplasm of inner and outer pillar cells, and labeling was also maintained in the inner spiral plexus and the auditory nerve fibers. It was interesting to note that immunofluorescence was present in the efferent tunnel crossing fibers, which crossed the tunnel of Corti at mid-level (Figure 3 A-D). Later, at the onset of hearing in mice (P12), ${ }^{24}$ the tunnel of Corti was wider, the apical surface process of the inner pillar cell is obviously bent over the outer pillar cell. At this time, acetylated tubulin displayed prominent and homogeneous labeling along the entire length of two cell types, and the foot plates resting on the basilar membrane exhibited strong labeling. Labeling for acetylated tubulin ran parallel to the apical surface of the outer pillar cell, forming the beam bundle. Linear labeling was present mainly in the Deiters' cell bodies, which defined the distance between the base of the OHCs and the basilar membrane, and the staining seemed to vanish from the OHCs synaptic region (Figure 3 E-F).
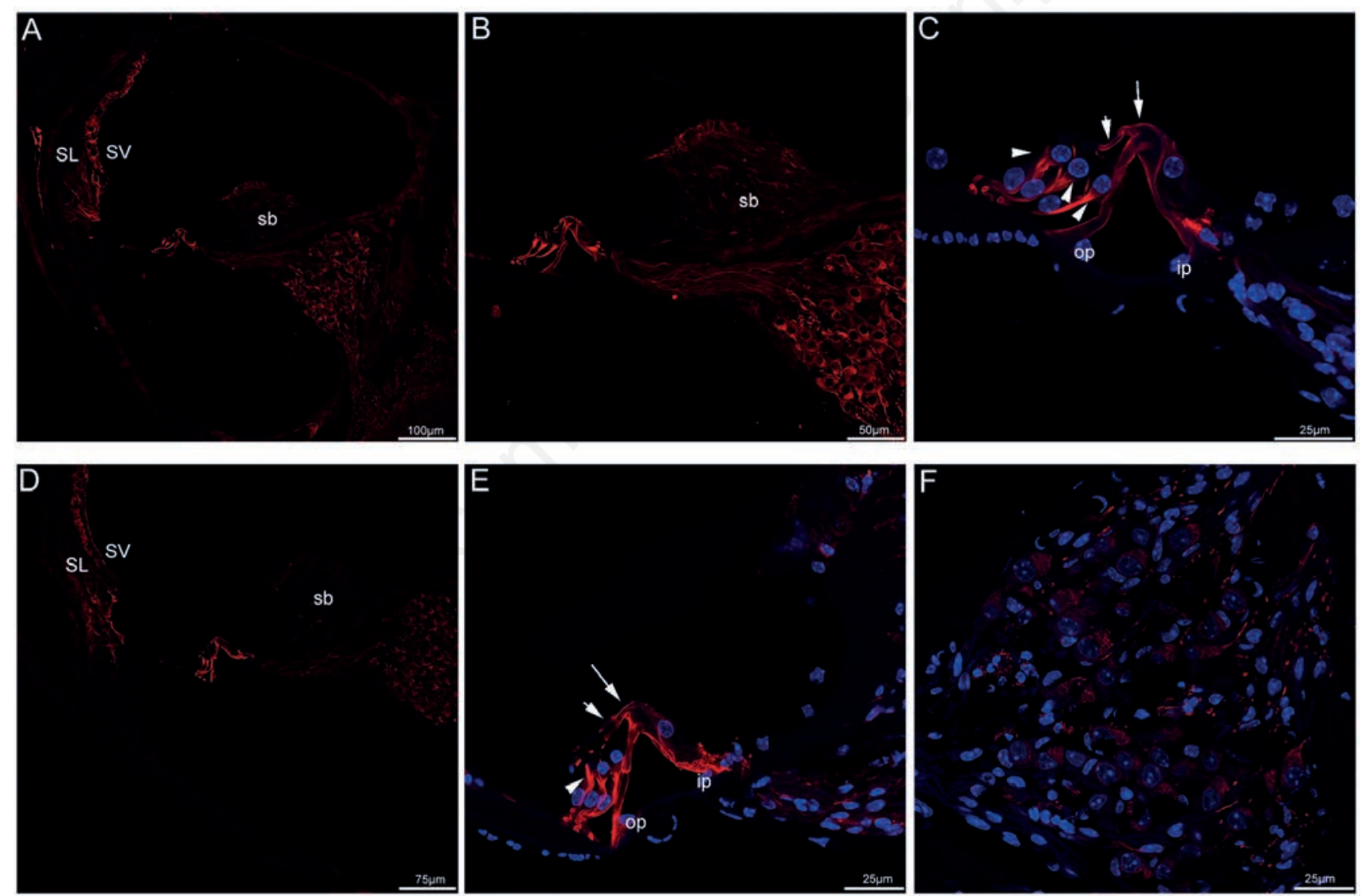

Figure 5. Acetylated tubulin immunolabeling in the mouse cochlea at P21 and P30. A) A low-magnification view of acetylated tubulin (red) labeling in the medial turn at P21. B) A high-magnification image of acetylated tubulin immunolabeling in the medial turn at P21. C) A higher-magnification image of acetylated tubulin immunolabeling in the organ of Corti in the medial turn at P21. Acetylated tubulin immunolabeling was prominent and uniform in both bundles in each of the pillar cells, the transcellular bundle (large arrow) and beam bundle (small arrow) showed positive immunostaining. Noted that acetylated tubulin immunolabeling in three rows of Deiters' cells was found at their cup-shaped middle regions (arrowheads). Merged image with cell nuclei stained with DAPI (blue). D) A low-magnification view of acetylated tubulin (red) labeling in the medial turn at P30. E) A higher-magnification image of acetylated tubulin immunolabeling in the organ of Corti in the medial turn at P30. Noted that acetylated tubulin continued to be expressed in Deiters' cups (arrowheads). Labeling appeared also in both transcellular bundle (large arrow) and the beam bundle (small arrow). Merged image with cell nuclei stained with DAPI (blue). F) Detail of acetylated tubulin (red) labeling in the Rosenthal's canal of the medial turn of P30. Acetylated tubulin showed no apparent labeling in the somata of spiral ganglion neurons. Cell nuclei were counterstained with DAPI (blue). SL, spiral ligament; SV, stria vascularis; sb, spiral limbus; op, outer pillar cell; ip, inner pillar cell. 
Expression of acetylated tubulin in mouse cochlea at P14, P17, P21 and the adult stage

Shortly after the onset of hearing (P14 and P17), the mouse cochlea morphologically reached an adult-like appearance, and the overall pattern of immunostaining was similar to that found in the adult mouse cochlea. Labeling for acetylated tubulin in the Deiters' cell was arranged in three parallel bundles. In the inner and outer pillar cells, and two bundles ran the full length of these two cell types, and acetylated tubulinlabeled beam bundle of outer pillar cell extended to interdigitate between the first row of OHCs. Note that labeling for acetylated tubulin formed transcellular bundle at apical surface of inner pillar cell. In addition, consistent with previous reports, in the cochlear lateral wall, immunofluorescence was also detected in the stria vascularis, root cell bodies, and a small number of fibrocytes of the spiral ligament. ${ }^{25}$ Strong staining in nerve terminals remained nearly unchanged (Figure 4 A-F). From P21 onward, acetylated tubulin exhibited more characteristic staining patterns. Three bundles were parallel to the longitudinal axis of Deiters' cell, and the specialized Deiters' cups developed, which held the base of the OHCs. In agreement with the concept that microtubules are long hollow cylinders, ${ }^{26}$ this study showed that two separate bundles ran throughout the entire length of each of the pillar cells, labeling for acetylated tubulin was distributed uniformly in the transcellular bundle (inner pillar cells) and beam bundle (outer pillar cells). In addition, expression of acetylated tubulin in the cochlear lateral wall and nerve terminals beneath the IHCs was also found (Figures 5 A-F and 6 A-F).

\section{Quantification of acetylated tubulin in pillar cells and Deiters' cells}

Specific regions on cryosections of the mouse cochlea (pillar cells and Deiters' cells) at P1, P5, P8, P12, P14, P17 and adult were selected as regions of interest, and the mean intensity was obtained from the fluorescence intensity histograms. In three rows of Deiters' cells, there were significant differences in the expression of acetylated tubulin between P5 compared with P10, $\mathrm{P} 14, \mathrm{P} 17$ and adult $(\mathrm{P}<0.05)$. There was no significant difference in the expression of acetylated tubulin between P5 compared
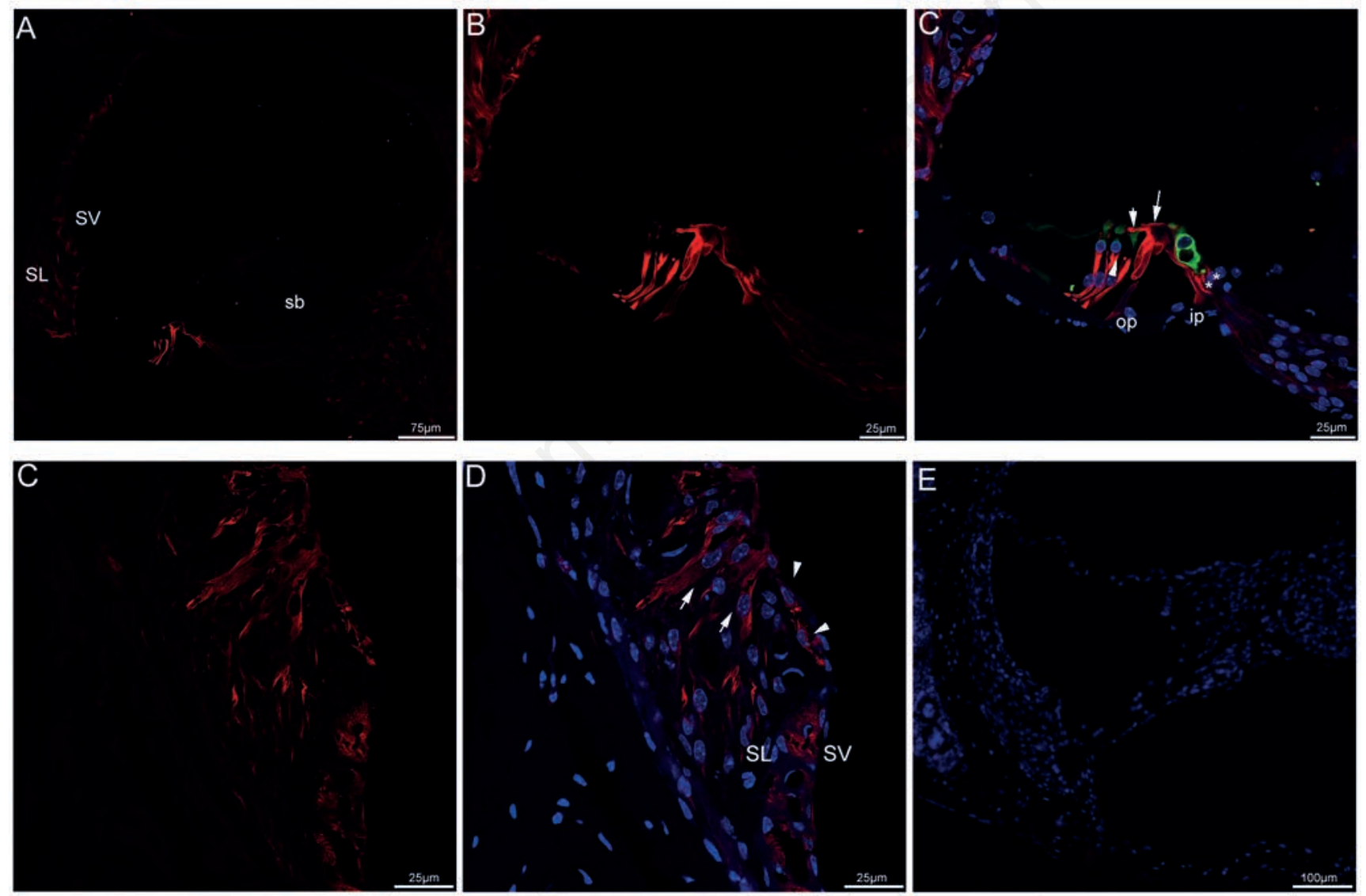

Figure 6. Acetylated tubulin immunolabeling in adult (P30+) mouse cochlea. A) An overview of acetylated tubulin (red) labeling in the medial turn of the mouse cochlea at adult stage. B,C) Detail of acetylated tubulin (red) labeling and myosin VIIa (green) labeling in the organ of Corti in the medial turn at adult stage and the merged image + DAPI. Acetylated tubulin immunolabeling in each of pillar cell ran from the apex toward the base, both transcellular bundle (large arrow) and the beam bundle (small arrow) continued to label acetylated tubulin. Noted that immunostaining continued to express in the nerve terminals (asterisks) under the inner hair cell. Immunostaining was also noted in Deiters' cups (arrowheads). Merged image with cell nuclei stained with DAPI (blue). D,E) Detail of acetylated tubulin labeling in the cochlear lateral wall. Acetylated tubulin expression could be seen in the root cells (arrowheads), root cell processes (arrows) and the stria vascularis. Cell nuclei were counterstained with DAPI (blue). F) The absence of acetylated tubulin and myosin VIIa immunofluorescence in negative control at adult mouse cochlea, omitting the primary antibody. Merged image with cell nuclei stained with DAPI (blue). SL, spiral ligament; SV, stria vascularis; sb, spiral limbus; op, outer pillar cell; ip, inner pillar cell 
with $\mathrm{P} 8$ and $\mathrm{P} 12(\mathrm{P}>0.05)$. In the pillar cells, there were significant differences in the expression of acetylated tubulin between P1 compared with P5, P8, P10, $\mathrm{P} 12, \mathrm{P} 14, \mathrm{P} 17$ and adult $(\mathrm{P}<0.05)$. There was no significant difference in the expression of acetylated tubulin between P5 compared with $\mathrm{P} 8, \mathrm{P} 17$ and adult $(\mathrm{P}>0.05)$.

\section{Discussion}

This study investigated the dynamic expression of acetylated tubulin in the postnatal developing cochlea of the mouse. We showed that expression of acetylated tubulin was initiated as early as P1, its expression was broadly distributed in mouse cochlea throughout postnatal maturation. This suggested that acetylated tubulin was essential for the postnatal development of mouse cochlea. At P1, acetylated tubulin immunolabeling was observed mainly in the nerve fibers and their synaptic area underneath the IHCs and OHCs. Subsequently, the neural stainings underneath the OHCs disappear with maturation. In contrast, we demonstrate, for the first time, that acetylated tubulin immunostaining persisted in nerve terminals under the IHCs throughout development and into adulthood. Given the fact that microtubules act as directional railways for organelle transport during synaptogenesis, ${ }^{27,28}$ we speculated that acetylated tubulin might be involved in the development and maintenance of cochlear innervation, through mediating intracellular transport of organelles.

Acetylated tubulin immunolabeling of the organ of Corti followed a precise spatiotemporal expression profile that was coincident with the development and maturation of the organ of Corti. Immunostaining for acetylated tubulin occurred firstly in the apex of pillar cells and proceeded basally. As with pillar cells, immunostaining in the Deiters' cells elongated downwards the base. Our findings further corroborated with previous reports in the gerbil that in the pillar and Deiters' cells, post-translational modifications progressed from cell apex to base in the same direction as microtubule elongation. ${ }^{14}$ Of particular interest was the change in the spatial extent and the intensity of immunolabeling across the pillar and Deiters' cells presented here that coincided temporally with the opening of the tunnel of Corti and spaces of Nuel. These fluid-filled spaces facilitate the sound wave that supports $\mathrm{OHC}$ amplification. ${ }^{29}$ These observations indicated that early postnatal acetylated tubulin expression participates in the development of pillar cells and the phalangeal processes of Deiters' cells and, as a result, in the formation of fluid spaces in the organ of Corti. Shortly after hearing onset to the adult, its expression profile exhibited only minor changes within the cochlear tis- sues, which might imply that developmentally regulated expression of acetylated tubulin correlated with the onset of hearing. In later postnatal stages, particularly strong immunolabelling was observed in pillar cells and three rows of Deiters' cells. Immunostaining in the pillar cells was arranged in parallel bundles which ran the entire length of each cell, and bundles were present in the bodies of Deiters' cell parallel to the long axis of the cells. Another interesting finding to come out of this study was the occurrence of acetylated tubulin immunoreactivity at mechanically important cell regions, namely the Deiters' cups, which played a critical role in the mechanical-coupling between Deiters' cells and OHCs. ${ }^{30}$ Our result supported the concept that microtubule bundles in supporting cells were stable and lent mechanical support to the organ of Corti in the late postnatal development of the cochlea, which was required for mechanical signals to mechano-sensitive hair cells..$^{31,32}$ In addition, in the lateral wall of the cochlea, stable localization of acetylated tubulin was observed in the stria vascularis, root cell bodies, and a small number of fibrocytes of the spiral ligament from P14 through into adulthood, which implied acetylated tubulin might be involved in recycling endolymphatic potassium that was required for normal cochlear functions. ${ }^{33}$

In conclusion, our data show that acetylated tubulin is broadly expressed in the
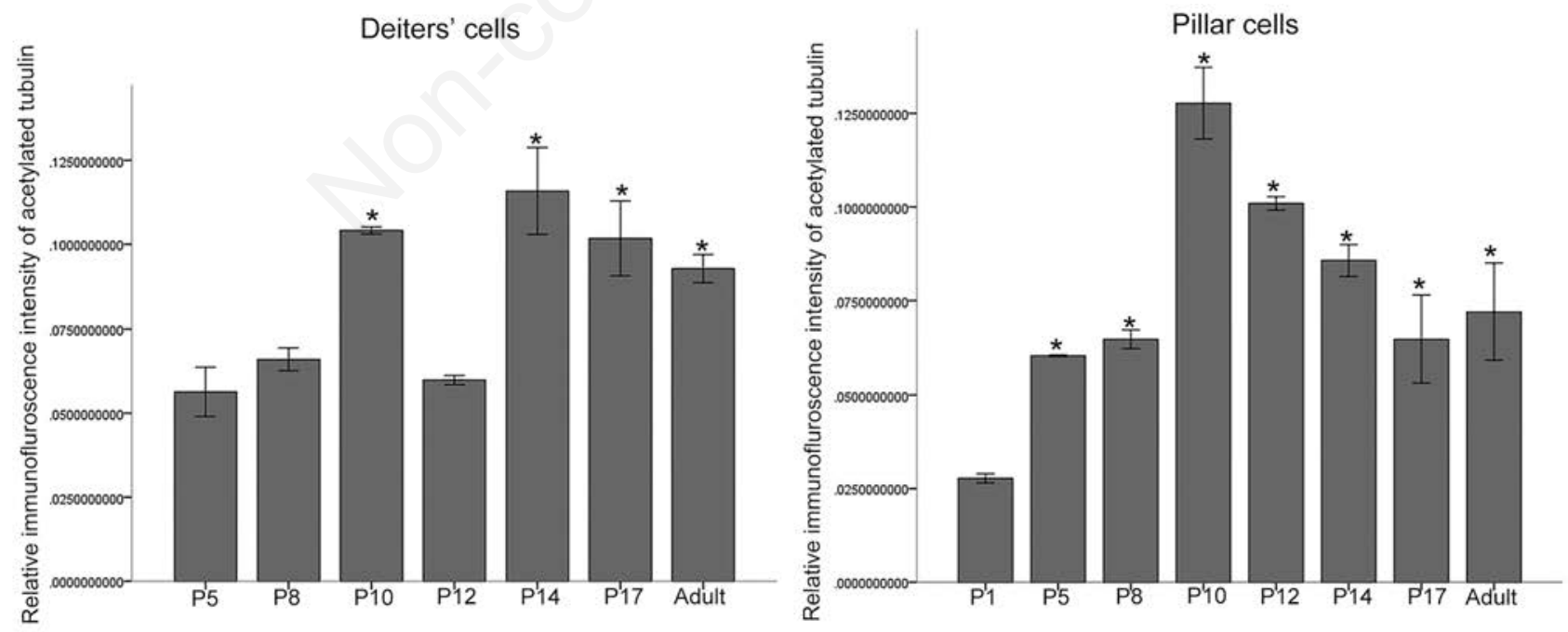

Figure 7. Graph representing the quantification of acetylated tubulin labeling intensity in the Deiters' cells and pillar cells of the organ of Corti during development. There were significant differences in the expression of acetylated tubulin in the Deiters' cells between P5 compared with P10, P14, P17 and adult $(\mathrm{P}<0.05)$. There was no significant difference in the expression of acetylated tubulin between P5 compared with P8 and P12 (P>0.05). There were significant differences in the expression of acetylated tubulin in the pillar cells between P1 compared with P5, P8, P10, P12, P14, P17 and adult $(\mathrm{P}<0.05)$. There was no significant difference in the expression of acetylated tubulin between $P 5$ compared with P8, P17 and adult $(P>0.05)$. 
developing mouse cochlea as early as postnatal day P1 and the distribution of acetylated tubulin appears to be developmentalstage dependent. Spatio-temporal expression pattern of acetylated tubulin in the mouse cochlea suggests its roles in postnatal cochlear development, auditory neurotransmission and cochlear micromechanics.

\section{References}

1. Akhshi TK, Wernike D, Piekny A. Microtubules and actin crosstalk in cell migration and division. Cytoskeleton (Hoboken) 2014;71:1-23.

2. Etienne-Manneville S. Microtubules in cell migration. Annu Rev Cell Dev Biol 2013;29:471-99.

3. Howes SC, Alushin GM, Shida T, Nachury MV, Nogales E. Effects of tubulin acetylation and tubulin acetyltransferase binding on microtubule structure. Mol Biol Cell 2014;25:257-66.

4. Aguilar A, Becker L, Tedeschi T, Heller $\mathrm{S}$, Iominic C, Maxence V. Nachury MV. A-tubulin K40 acetylation is required for contact inhibition of proliferation and cell-substrate adhesion. Mol Biol Cell 2014;25:1854-66.

5. Christopher P. Garnham, Antonina Roll-Mecak. The chemical complexity of cellular microtubules: tubulin posttranslational modification enzymes and their roles in tuning microtubule functions. Cytoskeleton (Hoboken) 2012; 69:442-63.

6. Li L, Yang XJ. Tubulin acetylation: responsible enzymes, biological functions and human diseases. Cell Mol Life Sci 2015;72:4237-55.

7. Badding MA, Dean DA. Highly acetylated tubulin permits enhanced interactions with and trafficking of plasmids along microtubules. Gene Ther 2013; 20:616-24.

8. Aguilar A, Becker L, Tedeschi T, Heller $\mathrm{S}$, Iomini C, Maxence V, Nachury MV et al. A-tubulin K40 acetylation is required for contact inhibition of proliferation and cell-substrate adhesion. Mol Biol Cell 2014;25:1854-66.

9. Bane BC, MacRae TH, Xiang H, Bateman J, Slepecky NB. Microtubule cold stability in supporting cells of the gerbil auditory sensory epithelium: correlation with tubulin post-translational modifications. Cell Tissue Res 2002; 307:57-67

10. Szarama KB, Gavara N, Petralia RS, Chadwick RS, Kelley MW. Thyroid hormone increases fibroblast growth factor receptor expression and disrupts cell mechanics in the developing organ of corti. BMC Dev Biol 2013;13:6.

11. Chen S, Xie L, Xu K, Cao HY, Wu X, Xu XX, et al. Developmental abnormalities in supporting cell phalangeal processes and cytoskeleton in the Gjb2 knockdown mouse model. Dis Model Mech 2018;11(2). pii: dmm033019

12. Saha S, Slepecky NB. Age-related changes in microtubules in the guinea pig organ of Corti. Tubulin isoform shifts with increasing age suggest changes in micromechanical properties of the sensory epithelium. Cell Tissue Res 2000;300:29-46.

13. Slepecky NB, Henderson CG, Saha S. Post-translational modifications of tubulin suggest that dynamic microtubules are present in sensory cells and stable microtubules are present in supporting cells of the mammalian cochlea. Hear Res 1995;91:136-47.

14. Tannenbaum J, Slepecky NB. Localization of microtubules containing posttranslationally modified tubulin in cochlear epithelial cells during development. Cell Motil Cytoskeleton 1997;38:146-62.

15. Yoshida A, Yamamoto N, Kinoshita M, Hiroi N, Hiramoto T, Kang G, et al. Localization of septin proteins in the mouse cochlea. Hear Res 2012;289:4051.

16. Liu WJ, Yang J. Preferentially regulated expression of connexin 43 in the developing spiral ganglion neurons and afferent terminals in post-natal rat cochlea. Eur J Histochem 2015;59:2464.

17. Liu WJ, Yang J. Developmental expression of inositol 1, 4, 5-trisphosphate receptor in the post-natal rat cochlea. Eur J Histochem 2015;59:2486.

18. Oesterle EC, Campbell S. Supporting cell characteristics in long-deafened aged mouse ears. J Assoc Res Otolaryngol 2009;10:525-44.

19. MacDonald GH, Rubel EW. Threedimensional imaging of the intact mouse cochlea by fluorescent laser scanning confocal microscopy. Hear Res 2008;243:1-10.

20. Magiera MM, Janke C. Investigating tubulin posttranslational modifications with specific antibodies. Methods Cell Biol 2013;115:247-67.

21. Fritzsch B, Fariñas I, Reichardt LF. Lack of neurotrophin 3 causes losses of both classes of spiral ganglion neurons in the cochlea in a region-specific fashion. J Neurosci 1997;17:6213-25.
22. Huang M, Kantardzhieva A, Scheffer D, Liberman MC, Chen ZY. Hair cell overexpression of Islet1 reduces age-related and noise-induced hearing loss. J Neurosci 2013;33:15086-94.

23. Souter M, Nevill G, Forge A. Postnatal maturation of the organ of Corti in gerbils: morphology and physiological responses. J Comp Neurol 1997;386: 635-51.

24. Huang LC, Greenwood D, Thorne PR, Housley GD. Developmental regulation of neuron-specific $\mathrm{P} 2 \mathrm{X} 3$ receptor expression in the rat cochlea. J Comp Neurol 2005;484:133-43.

25. Jagger DJ, Nevill G, Forge A. The membrane properties of cochlear root cells are consistent with roles in potassium recirculation and spatial buffering. J Assoc Res Otolaryngol 2010;11:435-48.

26. Horio T, Murata T. The role of dynamic instability in microtubule organization. Front Plant Sci 2014;5:511.

27. Rochlin MW, Wickline KM, Bridgman PC. Microtubule stability decreases axon elongation but not axoplasm production. J Neurosci 1996;16:3236-46.

28. Delépine C, Meziane H, Nectoux J, Opitz M, Smith AB, Ballatore C, et al. Altered microtubule dynamics and vesicular transport in mouse and human MeCP2-deficient astrocytes. Hum Mol Genet 2016;25:146-57.

29. Zagadou BF, Mountain DC. Analysis of the cochlear amplifier fluid pump hypothesis. J Assoc Res Otolaryngol 2012;13:185-97.

30. Parsa A, Webster P, Kalinec F. Deiters cells tread a narrow path--the Deiters cells-basilar membrane junction. Hear Res 2012;290:13-20.

31. Mellado Lagarde MM, Cox BC, Fang J, Taylor R, Forge A, Zuo J. Selective ablation of pillar and deiters' cells severely affects cochlear postnatal development and hearing in mice. $\mathrm{J}$ Neurosci 2013;33:1564-76.

32. Szarama KB, Gavara N, Petralia RS, Kelley MW, Chadwick RS. Cytoskeletal changes in actin and microtubules underlie the developing surface mechanical properties of sensory and supporting cells in the mouse cochlea. Development 2012;139:218797.

33. Locher H, de Groot JC, van Iperen L, Huisman MA. Development of the stria vascularis and potassium regulation in the human fetal cochlea: Insights into hereditary sensorineural hearing loss. Dev Neurobiol 2015;75:1219-40. 